\title{
„Wonneproppen“: \\ Deutsche Neugeborene deutlich schwerer als indische
}

\section{UKE-Wissenschaftler an WHO-Studie zum Geburtsgewicht beteiligt}

") Wie schwer ein Baby bei der Geburt ist, variiert von Land zu Land und wird von verschiedenen Faktoren wie dem Alter und dem Gewicht der Mutter, der Zahl der Geburten und dem Geschlecht des Kindes bestimmt. Das ist das Ergebnis einer multinationalen Studie der Weltgesundheitsorganisation (WHO Department for Human Reproductive Health), an der Wissenschaftler des Universitätsklinikums Hamburg-Eppendorf (UKE) maßgeblich beteiligt waren. So sind Kinder in Mittel- und Nordeuropa bei der Geburt deutlich schwerer als etwa in Indien oder im Kongo, wobei es sich jeweils um risikoarme Schwangerschaften handelt und die Frauen in einer vergleichbaren sozioökonomischen Umgebung leben.

Die Studie umfasste 1.387 gesunde Frauen aus zehn Ländern, darunter Ägypten, Argentinien, Kongo, Frankreich, Deutschland, Indien und Thailand. Studienzentrum für Deutschland war die Klinik für Geburtshilfe und Pränatalmedizin des UKE unter der
Leitung von Prof. Dr. Kurt Hecher. Die Neugeborenen der 139 in die Studie aufgenommenen Hamburger Mütter wogen im Durchschnitt 3.480 Gramm. Schwerer waren nur die norwegischen Babys mit durchschnittlich 3.575 Gramm. Am leichtesten waren die indischen Babys mit 2.975 Gramm. Da alle werdenden Mütter in einem vergleichbar guten Umfeld lebten, sind die Unterschiede nicht auf die jeweiligen sozioökonomischen Verhältnisse zurückzuführen.

In der Schwangerenvorsorge spielt die Ermittlung des wahrscheinlichen Geburtsgewichts eine wichtige Rolle, denn „ein geringes Geburtsgewicht ist mit einer höheren Sterblichkeit in der Geburtsphase, häufigeren Erkrankungen in der Kindheit und längerfristigen gesundheitlichen Risiken als Erwachsener verbunden“, erläutert Dr. Anke Diemert, Oberärztin in der UKEGeburtsklinik. Im Rahmen der Studie wurden nun anhand der Standard-Ultraschalluntersuchungen fetale Wachstumsdiagramme für den Kopf- und Bauchumfang, die Länge des Oberschenkelknochens und das Geburtsgewicht ermittelt. Schätzungen des Geburtsgewichts per Ultraschallmessungen sind ein wichtiges Instrument zur Identifizierung und Betreuung von Hochrisiko-Schwangerschaften. Dabei würden in vielen Ländern jedoch fetale Wachstumskurven verwendet, „die auf nur einer einzigen Population aus Ländern mit hohem Einkommen basieren“, so UKE-Studienleiter Hecher. Die vorliegende Studie mache die Unterschiede zwischen Ländern und Regionen deutlich.

Die WHO hat aufgrund dessen neue fetale Wachstumskurven entwickelt, die insbesondere die regionalen Unterschiede berücksichtigen und künftig in der Schwangerenvorsorge verwendet werden sollten. Sie sind online abrufbar und frei zugänglich. Die Ergebnisse der WHO-Untersuchung wurden in PLOS Medicine veröffentlicht.

www.uke.de

\section{Pränataldiagnostik - was ist wann sinnvoll?}

\section{Neue Versicherteninformation des G-BA zu vorgeburtlichen Untersuchungen auf dem Weg}

» Wie können genetisch bedingte Erkrankungen bereits während der Schwangerschaft erkannt werden, welche Untersuchungen gibt es und wann sind sie sinnvoll? Als Entscheidungshilfe für werdende Eltern plant der Gemeinsame Bundesausschuss

(G-BA) eine neue Versicherteninformation, die sich mit diesen Fragen beschäftigt. Die Deutsche Gesellschaft für Ultraschall in der Medizin e.V. (DEGUM) begrüßt das Vorhaben. Erblich bedingte Erkrankungen, Risikoschwangerschaften und -geburten können teilweise bereits bei Vorsorgeuntersuchungen erkannt werden. Ein Großteil dieser Untersuchungen wird per Ultraschall durchgeführt. „Die neue Versicherteninformation kann werdenden Eltern dabei helfen, die schwierige und persönliche Entscheidung zu treffen, welche der verfügbaren Untersuchungen sie in Anspruch nehmen möchten“, so Prof. Dr. med. Peter Kozlowski, Vorstandsmitglied der DEGUM. Da die Untersuchungen auf freiwilliger Basis erfolgen, sei es wichtig, „dass werdende Eltern gut aufgeklärt sind und im Falle einer Risikoschwangerschaft eine fundierte Entscheidung treffen können", betont auch Prof. Dr. med. Renaldo Faber, Vorsitzender der DEGUM-Sektion für Geburtshilfe und Gynäkologie

So sei es beispielsweise für Eltern wichtig zu wissen, welche Untersuchungen von der gesetzlichen Krankenkasse übernommen werden und welche nicht. So sind die frühe Feindiagnostik am Ende des ersten Drittels und der umfassende Organultraschall zwischen der 20. und 22. Schwangerschaftswoche keine Regelleistungen der gesetzlichen Krankenkassen. Da jedoch hier die meisten organischen, genetischen Anomalien bei Ungeborenen identifiziert werden können, plädiert Kozlowski dafür, dass die gesetzlichen Krankenkassen die Kosten für diese Untersuchungen im Rahmen der Mutterschafts-Richtlinien übernehmen. Darüber hinaus könnten bei diesen vorgeburtlichen Untersuchungen bei einer Mehrzahl der Frauen auch ernsthafte Komplikationen im Verlauf der Schwangerschaft erkannt werden.

www.degum.de 